\title{
Finding the position of a small body in the presence of a large body from scattering data
}

\author{
Yaakov Olshansky ${ }^{\mathrm{a} *}$ and Alexander G. Ramm ${ }^{\mathrm{b}}$ \\ ${ }^{a}$ Department of Applied Mathematics, Tel-Aviv University, Tel-Aviv, Israel; \\ ${ }^{b}$ Department of Mathematics, Kansas State University, Kansas, USA
}

(Received 16 January 2008; final version received 28 August 2008)

\begin{abstract}
The scattering problem by two bodies is considered. One of these bodies is small, the other one is large. The scattering problem for the large body is assumed solved. Under this assumption, the solution to the scattering problem on these two bodies is given analytically. This theory allows the solution of the inverse problem of finding the location of the small body and the electric capacitance of the perfect conductor with the shape of the small body from the scattering data of the two-body scattering problem. To test the theory, the capacitance of the small body is calculated from the scattering data by the developed theory and then compared with the known exact value of the capacitance. A numerical simulation for scattering by two spheroids is analysed. A good agreement between the numerical and the analytical results is found.
\end{abstract}

Keywords: two-body scattering problem; electric capacitance; scattering by small body

AMS Subject Classifications: 78A46; 81U40

\section{Wave scattering by two bodies}

Let us assume that one body $D_{1}$ is not small in comparison with the wavelength $\lambda$, and the other body $D_{2}$ is small, that is, $k a \ll 1$, where $a=0.5 \operatorname{diam} D_{2}, k=2 \pi / \lambda$ is the wavenumber. The scattering problem consists of finding the scattering solution $u=u(x, \alpha, k)$ such that

$$
\begin{gathered}
\left(\nabla^{2}+k^{2}\right) u=0 \quad \text { in } \mathbb{R}^{3} \backslash D, \quad D:=D_{1} \cup D_{2}, \\
\left.u\right|_{S}=0, \quad S=S_{1} \cup S_{2}, \quad S_{j}=\partial D_{j}, \quad j=1,2, \\
u=u_{0}+v, \quad u_{0}:=e^{i k \alpha \cdot x}, \quad \alpha \in S^{2}, \\
v=A(\beta, \alpha, k) \frac{e^{i k r}}{r}+o\left(\frac{1}{r}\right), \quad r:=|x| \rightarrow \infty, \quad \frac{x}{r}=\beta .
\end{gathered}
$$

*Corresponding author. Email: oyakov@post.tau.ac.il 
Here $S^{2}$ is the unit sphere, $\alpha$ the direction of the incident plane wave (incident direction) and $\beta$ is the direction of the scattered wave, $\alpha, \beta \in S^{2}$. The coefficient $A(\beta, \alpha, k)$ is called the scattering amplitude. It is well known that problem (1)-(4) has a unique solution (see e.g. [1]). We do not assume necessarily that $D_{1}$ and $D_{2}$ are connected. The following assumptions are important for our theory:

(1) $k a \ll 1$; if $D_{2}$ consists of several small bodies, then the union $D_{2}$ of these bodies should have small diameter $2 a$, such that $k a \ll 1$, and

(2) $k d \gg 1$, i.e. the small body $D_{2}$ is located in the far-zone from the large body. Here $d$ is the distance between $D_{1}$ and $D_{2}$.

The usual way to calculate the solution to (1)-(4) is to use the boundary integral equations. Although the amount of literature on many-body wave scattering is large (see [2] and references therein), there are very few numerical results, and the published results do not include the case of wave scattering by large and small bodies. Our article presents an asymptotically rigorous treatment of wave scattering in this case.

In this article, we assume that the scattering problem for a single perfectly conducting body $D_{1}$ is solved, and solve the scattering problem for two bodies $D_{1}$ and $D_{2}$ assuming $D_{2}$ small. Under the basic assumptions (1) and (2), we bypass the numerical solution of integral equations, and obtain asymptotic formulas for the scattered field with a negligible error if the parameter $k a$ is sufficiently small and the parameter $k d$ is sufficiently large. We give a method for finding an unknown position of the small body from the scattering data. If the body $D_{2}$ is small, its position can be characterized by one vector $\xi$. Moreover, we estimate from the scattering data some parameter (electric capacitance of a perfect conductor with the shape of the small body), which is of the order of the size $a$ of the small body. We give the order of the error of the analytical formulas for the scattered field. It is known [1], that the scattering amplitude known at a fixed $k>0$ for all incident directions $\alpha$ and all scattering directions $\beta$ determines the shapes of the obstacles uniquely as well as the boundary conditions of the Dirichlet, Neumann, or Robin type on the surface of these obstacles.

Denote by $u_{1}$ the solution of the scattering problem for the body $D_{1}$ in the absence of the small body $D_{2}$. The exact solution of the scattering problem (1)-(4) can be written as:

$$
u(x)=u_{1}(x)+\int_{S_{2}} g_{1}(x, t) \sigma(t) \mathrm{d} t
$$

where

$$
\left(\nabla^{2}+k^{2}\right) g_{1}(x, y)=-\delta(x-y) \quad \text { in } \mathbb{R}^{3} \backslash D_{1},
$$

$g_{1}=0$ on $S_{1}$ and satisfies the radiation condition. For any $\sigma$ the function $u$ in (5) satisfies Equations (1), (3), (4), and the boundary condition (2) on $S_{1}$. If $\sigma$ is chosen so that (2) holds also on $S_{2}$, then the function $u$ in (5) satisfies Equations (1)-(4). Since the solution to problem (1)-(4) is unique, formula (5) yields this solution if $\sigma$ is chosen so that boundary condition (2) is satisfied. This argument does not depend on the assumption about the smallness of $D_{2}$.

In [1, p. 46], the following formula is established:

$$
g_{1}(\xi, y)=\frac{e^{i k|y|}}{4 \pi|y|} u_{1}(\xi,-\beta)+O\left(\frac{1}{|y|^{2}}\right), \quad|y| \rightarrow \infty, \quad \beta:=\frac{y}{|y|} .
$$


Here $\xi$ can be any point in any fixed bounded region.

If $D_{2}$ is small and the distance $d$ between $D_{1}$ and $D_{2}$ is large, $k d>>1$, d $\gg$ diam $D_{1}$, so that $D_{2}$ is located in the far zone of $D_{1}$, and if $|x-\xi|>>a$, then one can write:

$$
u(x, \alpha)=u_{1}(x, \alpha)+g_{1}(x, \xi) Q+O\left(\frac{a}{d}\right), \quad Q:=\int_{S_{2}} \sigma(t) \mathrm{d} t, \quad k d>>1 .
$$

Here and below $\xi \in D_{2}$ is an arbitrary fixed point, and our goal is to estimate $\xi$ from the scattering data. If this is done, then the position of $D_{2}$ is estimated with the error of the order of $a$.

An estimate of the error term $O(a / d)$ is obtained as follows:

$$
\left|\int_{S_{2}}\left[g_{1}(x, t)-g_{1}(x, \xi)\right] \sigma(t) \mathrm{d} t\right| \leq c\left|\nabla g_{1}(x, \xi)\right| a|Q|=O\left(\frac{a}{d}\right) .
$$

We have used the estimate $\left|\nabla g_{1}(x, \xi)\right| \leq c \max \left((k / d),\left(1 / d^{2}\right)\right)$, which is valid if $k|x-\xi|>>1$. We took into account that $k a \ll 1$ and $Q=O(a)$, see formula (12) below. The electric capacitance $C$ of the perfect conductor with the shape of the small body $D_{2}$ is of the order of the size of $D_{2}$, that is, $C=O(a)$.

From (6) and (7) one derives the following formula for the scattering amplitude: $A(\beta, \alpha)$ for the two-body scattering problem:

$$
A(\beta, \alpha)=A_{1}(\beta, \alpha)+\frac{1}{4 \pi} u_{1}(\xi,-\beta) Q:=A_{1}(\beta, \alpha)+A_{2}(\beta, \alpha),
$$

where $|x| \rightarrow \infty, \beta:=x /|x|$, and $A_{1}$ is the scattering amplitude for the scattering problem for one body $D_{1}$ in the absence of the second body and for the incident plane wave $e^{-i k \beta \cdot \xi}$.

We assume that the conditions $k d>>1, k a \ll 1,|x-\xi|>>a$ are satisfied. In this case one can derive an exact formula for the scattering amplitude:

$$
A(\beta, \alpha)=A_{1}(\beta, \alpha)-\frac{C}{4 \pi} u_{1}(\xi, \alpha) u_{1}(\xi,-\beta):=A_{1}(\beta, \alpha)+A_{2}(\beta, \alpha)
$$

We use in the numerical calculations the following consequence of the above exact formula, namely, the following approximate formula for the scattering amplitude:

$$
A(\beta, \alpha)=A_{1}(\beta, \alpha)-\frac{C}{4 \pi} u_{1}(\xi, \alpha) e^{-i k \beta \cdot \xi} .
$$

This formula is the more accurate the larger the parameter $k d$ is. We have used above the following formula for $Q$ :

$$
Q=-\frac{C}{4 \pi} u_{1}(\xi, \alpha)
$$

and an approximate formula for $u_{1}(\xi,-\beta)$, which is valid if the small body $D_{2}$ is located in the far zone of the large body $D_{1}$, that is, if $k d>>1$ :

$$
u_{1}(\xi,-\beta)=e^{-i k \beta \cdot \xi}+O\left(\frac{1}{k d}\right), \quad d=|x-\xi| .
$$


Let us derive formula (12). The boundary condition on $S_{2}$ and (5) yield

$$
-u_{1}(s)=\int_{S_{2}} g_{1}(s, t) \sigma(t) \mathrm{d} t .
$$

One can find $\sigma$ uniquely from this equation, which is uniquely solvable because $k^{2}$ is not a Dirichlet eigenvalue of the Laplacian in $D_{2}$, due to the assumption that $D_{2}$ is small. Our theory can be generalized to the case of other boundary conditions because in [3] formulas for the scattering amplitude for a small body are derived for Neumann and Robin boundary conditions, and not only for the Dirichlet conditions, but we do not go into further detail here.

If the body $D_{2}$ is small and is far away from $D_{1}$, i.e. $k d>>1$, then

$$
u_{1}(s)=u_{1}(\xi)+O(a)
$$

and one derives an asymptotic formula

$$
g_{1}(s, t)=\frac{1}{4 \pi|s-t|}(1+O(|s-t|), \quad \text { as }|s-t| \rightarrow 0 .
$$

Thus, with a negligible error, Equation (14) for $\sigma$ can be written as

$$
-u_{1}(\xi)=\int_{S_{2}} \frac{1}{4 \pi|s-t|} \sigma(t) \mathrm{d} t .
$$

The error can be estimated as follows: $\left|u_{1}(\xi)-u_{1}(s)\right| \leq|\nabla u(y) \| \xi-s|=O(a / d)$, where $y$ is some point on the segment joining points $s$ and $\xi$, and the error of replacing $g_{1}(s, t)$, where $s, t \in S_{2}$, is $O(k a)$.

Equation (17) is an equation for the electrostatic charge density $\sigma$ on the surface $S_{2}$ of a perfect conductor $D_{2}$, charged to a constant potential $-u_{1}(\xi)$. The total charge is

$$
Q=\int_{S_{2}} \sigma(t) \mathrm{d} t=\frac{1}{4 \pi} C\left(-u_{1}(\xi, \alpha)\right),
$$

where $C$ is the electric capacitance of the perfect conductor with the shape $D_{2}$, and the factor $1 / 4 \pi$ normalizes the capacitance so that the capacitance of a ball of radius $a$ equals to $a$. Thus, formula (12) is derived. One could have looked for $u$ of the form (5) with $g_{1}$ replaced by

$$
g:=g(x, t):=\frac{e^{i k|x-t|}}{4 \pi|x-t|},
$$

and obtain formula (11) again, but less rigorously because with the above replacement the function $u$ would satisfy the boundary condition on $S_{1}$ approximately, with the error $O(a / d)$, rather than exactly.

Let us give some estimates for $g$. These estimates show that the far zone for a small body starts already at the distances $\rho>a$, while for the body, which is not small, the far zone starts at the distances $\rho>>\lambda$, which is much further than for small bodies. This follows from the inequality

$$
\frac{k a^{2}}{\rho}=k a \cdot \frac{a}{\rho}<k a<<1 .
$$


If $k a \ll 1$ and $|x-\xi|>\rho \gg a$, then for $t \in S_{2}$

$$
g(x, t)=g(x, \xi)\left(1+O\left(k a+\frac{a}{\rho}\right)\right) .
$$

Indeed

$$
|x-t|=|x-\xi-(t-\xi)|=|x-\xi|\left(1+O\left(\frac{a}{\rho}\right)\right)
$$

and

$$
\begin{aligned}
k|x-t| & =k|x-\xi| \sqrt{1-2 \frac{((x-\xi /|x-\xi|), t-\xi)}{|x-\xi|}+\frac{|t-\xi|^{2}}{|x-\xi|^{2}}} \\
& =k|x-\xi|-k\left(\frac{x-\xi}{|x-\xi|}, t-\xi\right)+O\left(\frac{k a^{2}}{\rho}\right) .
\end{aligned}
$$

In our formula (11) the capacitance $C$ appears. Analytic formulas for the capacitances of conductors of arbitrary shapes are given in [3, p. 26]. These formulas allow one to calculate $C$ with any desired accuracy. In (11) the $k$-dependence in $A$ and $A_{1}$ is not shown because $k>0$ is fixed. We assume $u_{1}(x, \alpha)$ and $A_{1}(\beta, \alpha)$ known, because we have assumed that the scattering problem for body $D_{1}$ in the absence of body $D_{2}$ is solved. Therefore, if $\xi$ is known, then the right-handside of (11) is known.

Consider the inverse problem of finding $\xi$, i.e. finding the position of the small body, and of $C$, its electric capacitance, from the knowledge of $A(\beta, \alpha)$ at a fixed $\alpha$. Since $A_{1}$ is known, the quantity

$$
a(\alpha, \beta):=C u_{1}(\xi, \alpha) e^{-i k \beta \cdot \xi}
$$

is known. If $\beta_{1} \neq \beta_{2}$ and $u_{1}(\xi, \alpha) \neq 0$, then

$$
\frac{a\left(\alpha, \beta_{1}\right)}{a\left(\alpha, \beta_{2}\right)}=e^{-i k\left(\beta_{1}-\beta_{2}\right) \cdot \xi} .
$$

Denote $\beta_{1}-\beta_{2}=\gamma$. One has

$$
\ln \frac{a\left(\alpha, \beta_{1}\right)}{a\left(\alpha, \beta_{2}\right)}=-i k \gamma \cdot \xi+2 \pi i n,
$$

where $n$ is an integer. Let $\gamma_{1}=\left(\beta_{1}-\beta_{2}\right) / 2$ and $v=\left(\beta_{1}+\beta_{2}\right) / 2$. Then $a\left(\alpha, \beta_{1}\right)=$ $a\left(\alpha, \gamma_{1}+v\right):=a\left(\alpha, \gamma_{1}, \nu\right), a\left(\alpha, \beta_{2}\right)=a\left(\alpha,-\gamma_{1}-v\right):=a\left(\alpha,-\gamma_{1},-v\right)$. Thus, one gets:

$$
\xi=-\frac{1}{i k} \nabla_{\gamma} \ln \frac{a\left(\alpha, \beta_{1}\right)}{a\left(\alpha, \beta_{2}\right)}=-\frac{1}{2 i k} \nabla_{\gamma_{1}} \ln \frac{a\left(\alpha, \gamma_{1}, \nu\right)}{a\left(\alpha,-\gamma_{1},-\nu\right)} .
$$

Stable differentiation formulas of noisy functions are given in [1], Appendix 4, and in [4], Chapter 13. From the definition of $a(\alpha, \beta)$ one gets:

$$
C=\frac{a(\alpha, \beta) e^{i k \beta \cdot \xi}}{u_{1}(\xi, \alpha)}
$$


The above theory assumes that the scattering amplitude $A$ is measured without noise. If $A$ is measured with some noise level $\delta$, then the quantity $a(\alpha, \beta):=a(\beta)$ is not known, but $a_{\delta}(\beta)$ is known such that

$$
\sup _{\beta \in S^{2}}\left|a_{\delta}(\beta)-a(\beta)\right| \leq \delta .
$$

One has

$$
\frac{a_{\delta}\left(\beta_{1}\right)}{a_{\delta}\left(\beta_{2}\right)}=\frac{a\left(\beta_{1}\right)}{a\left(\beta_{2}\right)}\left[1+O\left(\frac{\delta}{C\left|u_{1}(\xi, k)\right|}\right)\right] .
$$

The quantity $\left|u_{1}(\xi, k)\right|$ is assumed to be much larger than $\delta$. For example, $\left|u_{1}(\xi, k)\right| \approx 1$ if $\operatorname{dist}\left(\xi, D_{1}\right) \gg 1$ because

$$
u_{1}=u_{0}+O\left(\frac{1}{|x|}\right), \quad|x| \rightarrow \infty, \quad \text { and } \quad\left|u_{0}\right|=1 .
$$

Thus, the error term in (29) is $O(\delta / a)$ since $C=O(a)$. Roughly speaking, if $\delta / a<0.05$, then one can locate the position $\xi$ of $D_{2}$ relatively accurately using the above scheme. Our theory is an asymptotic one with $k a$ a small parameter and $k d$ a large parameter. The error of the theory tends to zero when $k a \rightarrow 0$ and $k d \rightarrow \infty$. In practice one can always treat the scattering data as noisy data.

\section{Scattering by a small and large prolate spheroids}

Let $S_{1}$ and $S_{2}$ be two prolate spheroids one of which is small compared with the wavelength, while the other is of the order of the wavelength or larger. Our aim is to find the location of the small prolate spheroid, i.e. the point $\xi=\left(\xi_{1}, \xi_{2}, \xi_{3}\right)$ in (24), and to calculate its capacitance (28), given the scattering amplitude for the two-body scattering problem. These two bodies are the small and the large spheroids.

The theory developed in this article can be generalized for the case of electromagnetic (EM) wave scattering. Every small body scatters EM waves as a suitable ellipsoid. For scalar wave scattering by a small acoustically soft body the scattering is isotropic and the scattering amplitude is determined by one scalar parameter, which is of the order $O(a)$. This parameter is the electric capacitance $C$ of the conductor with the shape of small body [3].

Let us introduce two coordinate systems: the prolate spheroidal coordinates $(\zeta, \theta, \varphi)$ and the rectangular Cartesian coordinates $(x, y, z)$. The relation between coordinate systems is given by the formulas:

$$
\begin{aligned}
& x=f \sinh \zeta \sin \theta \cos \varphi, \\
& y=f \sinh \zeta \sin \theta \sin \varphi, \\
& z=f \cosh \zeta \cos \theta,
\end{aligned}
$$

where $0 \leq \zeta<\infty, 0 \leq \theta \leq \pi, 0 \leq \varphi<2 \pi$. The $\zeta$ remains constant on the surface of the confocal prolate spheroid with semi-major axis $a=f \cosh \zeta$ and semi-minor axis $b=f \sinh \zeta$ and semi-focal distance $f=\sqrt{a^{2}-b^{2}}$.

Let us consider the plane wave with the incident direction $\alpha$ :

$$
u_{0}=e^{i k(x \sin \tilde{\alpha}+z \cos \tilde{\alpha})}=e^{i k f(\sinh \zeta \sin \theta \cos \varphi \sin \tilde{\alpha}+\cosh \zeta \cos \theta \cos \tilde{\alpha})} .
$$


The scattered field solution for prolate spheroid written in the prolate spheroidal coordinate system is given by [5, p. 422]:

$$
\begin{aligned}
u_{s}= & -2 \sum_{m=0}^{\infty} \sum_{n=m}^{\infty} \epsilon_{m} \frac{i^{n}}{N_{m n}} \frac{R_{m n}^{(1)}\left(k f, \cosh \zeta_{1}\right)}{R_{m n}^{(3)}\left(k f, \cosh \zeta_{1}\right)} \\
& \times R_{m n}^{(3)}(k f, \cosh \zeta) S_{m n}(k f, \cos \widetilde{\alpha}) S_{m n}(k f, \cos \theta) \cos m \varphi,
\end{aligned}
$$

with $\epsilon_{0}=1, \epsilon_{m}=2$ for all $m \geq 1, N_{m n}$ are the normalizing constants, $\zeta_{1}$ is the coordinate of the surface of the large prolate spheroid, $R_{m n}^{(1)}\left(k f, \zeta_{1}\right), R_{m n}^{(3)}\left(k f, \zeta_{1}\right)$ radial prolate function, $S_{m n}(k f, \cos \widetilde{\alpha})$ angular prolate function, $(\zeta, \theta, \varphi)$ is the scattering direction, $u_{s}$ is the scattered field, $u_{s}=u_{1}-u_{0}, u_{0}$ is the incident field, and $u_{1}$ is the scattering solution, i.e. the solution to the scattering problem for the large spheroid.

The origin of the coordinate system is chosen at the centre of a large prolate spheroid, and the small prolate spheroid is assumed to be located at some unknown distance $d$ from the large prolate spheroid, $k d>>1$.

The scattering amplitude for a small prolate spheroid is given in [5, p. 422]:

$$
\begin{aligned}
A_{2}\left(\left(\zeta_{2}, \theta, \varphi\right), \alpha\right)= & 2 i \sum_{m=0}^{\infty} \sum_{n=m}^{\infty} \epsilon_{m} \frac{i^{n}}{N_{m n}} \frac{R_{m n}^{(1)}\left(k f, \cosh \zeta_{2}\right)}{R_{m n}^{(3)}\left(k f, \cosh \zeta_{2}\right)} \\
& \times S_{m n}(k f, \cos \widetilde{\alpha}) S_{m n}(k f, \cos \theta) \cos (m \varphi) .
\end{aligned}
$$

From Equation (25) one gets the following relation:

$$
\arg \frac{a\left(\beta_{1}\right)}{a\left(\beta_{2}\right)}=-k\left(\beta_{1}-\beta_{2}\right) \cdot \xi-2 \pi n=-k \sum_{i=1}^{3} \gamma_{i} \xi_{i}-2 \pi n .
$$

Equation (25) is an equation for the three unknown components of the vector $\xi$. We use this equation with three different choices of $\gamma_{j}$ for finding $\xi$ as a solution of a linear algebraic system. In general, an integer number $n$ is unknown. We assume below that $n=0$ in (33) and solve the linear system (35), and then test that (33) is valid with $n=0$. Below we refer to this method as solving the linear system (Section 2.1).

When the difference between $\beta_{1}$ and $\beta_{2}$ is sufficiently small one may take $n=0$. In the limit $\beta_{1}-\beta_{2} \rightarrow 0$ one gets formula (27). The results based on formula (27) are presented in the last section and in Section 2.2 an application of the stable differentiation method is explained.

\subsection{Finding $\xi$}

In Cartesian coordinate system the scattering direction is given by: $\beta=(b \sin \theta \cos \varphi$, $b \cos \theta \sin \varphi, a \cos \theta)$. In order to find $\xi=\left(\xi_{1}, \xi_{2}, \xi_{3}\right)$ using (33), we consider a few scattering directions $\beta_{j}$, which are chosen so that the condition number of the matrix $\Gamma$ (see below) is not large (in our example this number is $\kappa(\Gamma)=10.55$ ):

$$
\begin{aligned}
& \beta_{1}=\left(b \sin \frac{\pi}{180} \cos \frac{80 \pi}{180}, b \cos \frac{\pi}{180} \sin \frac{8 \pi}{180}, a \cos \frac{\pi}{180}\right) \\
& \beta_{2}=\left(b \sin \frac{7 \pi}{180} \cos \frac{70 \pi}{180}, b \cos \frac{7 \pi}{180} \sin \frac{70 \pi}{180}, a \cos \frac{7 \pi}{180}\right)
\end{aligned}
$$




$$
\begin{aligned}
& \beta_{3}=\left(b \sin \frac{15 \pi}{180} \cos \frac{85 \pi}{180}, b \cos \frac{15 \pi}{180} \sin \frac{85 \pi}{180}, a \cos \frac{15 \pi}{180}\right) \\
& \beta_{4}=\left(b \sin \frac{75 \pi}{180} \cos \frac{75 \pi}{180}, b \cos \frac{75 \pi}{180} \sin \frac{75 \pi}{180}, a \cos \frac{75 \pi}{180}\right) \\
& \beta_{5}=\left(b \sin \frac{25 \pi}{180} \cos \frac{25 \pi}{180}, b \cos \frac{25 \pi}{180} \sin \frac{25 \pi}{180}, a \cos \frac{25 \pi}{180}\right) .
\end{aligned}
$$

This leads to the following linear system for finding $\xi$ :

$$
\left\{\begin{array}{c}
-\frac{1}{k} \arg \frac{a\left(\beta_{3}\right)}{a\left(\beta_{1}\right)}=\gamma_{1}^{(1)} \xi_{1}+\gamma_{2}^{(1)} \xi_{2}+\gamma_{3}^{(1)} \xi_{3} \\
-\frac{1}{k} \arg \left(\frac{a\left(\beta_{4}\right)}{a\left(\beta_{2}\right)}\right)=\gamma_{1}^{(2)} \xi_{1}+\gamma_{2}^{(2)} \xi_{2}+\gamma_{3}^{(2)} \xi_{3} \\
-\frac{1}{k} \arg \left(\frac{a\left(\beta_{5}\right)}{a\left(\beta_{3}\right)}\right)=\gamma_{1}^{(3)} \xi_{1}+\gamma_{2}^{(3)} \xi_{2}+\gamma_{3}^{(3)} \xi_{3},
\end{array}\right.
$$

where

$$
\left(\begin{array}{l}
\beta_{3}-\beta_{1} \\
\beta_{4}-\beta_{2} \\
\beta_{5}-\beta_{3}
\end{array}\right)=\left(\begin{array}{l}
\gamma^{(1)} \\
\gamma^{(2)} \\
\gamma^{(3)}
\end{array}\right)
$$

or, in a matrix form,

$$
\Gamma\left(\begin{array}{l}
\xi_{1} \\
\xi_{2} \\
\xi_{3}
\end{array}\right)=\left(\begin{array}{c}
-\frac{1}{k} \arg \frac{a\left(\beta_{3}\right)}{a\left(\beta_{1}\right)} \\
-\frac{1}{k} \arg \frac{a\left(\beta_{4}\right)}{a\left(\beta_{2}\right)} \\
-\frac{1}{k} \arg \frac{a\left(\beta_{5}\right)}{a\left(\beta_{3}\right)}
\end{array}\right),
$$

where

$$
\Gamma:=\left(\begin{array}{lll}
\gamma_{1}^{(1)} & \gamma_{2}^{(1)} & \gamma_{3}^{(1)} \\
\gamma_{1}^{(2)} & \gamma_{2}^{(2)} & \gamma_{3}^{(2)} \\
\gamma_{1}^{(3)} & \gamma_{2}^{(3)} & \gamma_{3}^{(3)}
\end{array}\right) .
$$

For the solution of linear system to be stable with respect to small perturbations of the data, the matrix $\Gamma$ should be well-conditioned, i.e. its condition number $\kappa(\Gamma):=\|\Gamma\|\left\|\Gamma^{-1}\right\|$ should not be too large.

The condition number of $\Gamma$ depends on the chosen scattering directions. For the directions, chosen by us, the condition number is $\kappa(\Gamma)=10.55$. Therefore, the matrix $\Gamma$ is well-conditioned. It is shown in Section 3 by numerical experiments that small variations of the right-hand side of (35) lead to small changes in the solution $\xi$.

Since our matrix $\Gamma$ is of small size, it is practically not difficult to evalute its condition number for any given directions, and choose directions for which $\kappa(\Gamma)$ is not large. If this is done, there is no need to use a regularization for solving system (35). 
From (11) the capacitance $C$ is calculated by the formula:

$$
C=\frac{-4 \pi e^{i k \beta \cdot \xi}\left(A(\beta, \alpha)-A_{1}(\beta, \alpha)\right)}{u_{1}(\xi, \alpha)} .
$$

In order to calculate $u_{1}(\xi, \alpha)$ one returns to prolate coordinate system because $\xi$ is calculated in Cartesian coordinate system while formulas (30) and (31) for $u_{1}(\xi, \alpha)$ are given in the prolate spheroidal coordinate system.

\subsection{Calculating the derivative}

This section provides practical recommendations for using formula (27) when the data are noisy.

In the presence of noise formula (27) can be written as:

$$
\xi=-\frac{1}{2 i k} \nabla_{\gamma_{1}} \ln \frac{a\left(\alpha, \gamma_{1}, \nu\right)+\delta}{a\left(\alpha,-\gamma_{1}, \nu\right)+\delta},
$$

where $\delta$ is the noise level [3, p. 344].

The noisy function $\ln \left[\left(\left(a\left(\alpha, \gamma_{1}, v\right)+\delta\right) /\left(a\left(\alpha,-\gamma_{1}, v\right)+\delta\right)\right)\right]$ may be written as:

$$
\ln \frac{a\left(\alpha, \gamma_{1}, \nu\right)}{a\left(\alpha,-\gamma_{1}, \nu\right)}+\ln \frac{1+\left(\delta / a\left(\alpha, \gamma_{1}, \nu\right)\right)}{1+\left(\delta / a\left(\alpha,-\gamma_{1}, \nu\right)\right)}:=f+n_{\delta}:=f_{\delta},
$$

where $f$ is the exact data, $n_{\delta}$ is the noise, and $f_{\delta}$ is the noisy data, observed experimentally.

According to the theory of stable numerical differentiation [3, p. 344], the derivative of $f$ is estimated from the noisy data $f_{\delta}$ by the formula:

$$
f^{\prime}:=f_{\gamma_{1}}^{\prime} \approx \frac{f_{\delta}\left(\gamma_{1}+h\right)-f_{\delta}\left(\gamma_{1}-h\right)}{2 h}
$$

where the step $h=h(\delta)$ should be chosen by the formula:

$$
h=\sqrt{\frac{2 \delta}{M}}, \quad M=\max _{\gamma_{1}}\left|f^{\prime \prime}\right|,
$$

and

$$
f^{\prime \prime}\left(\gamma_{1}\right)=\frac{a^{\prime \prime}\left(\gamma_{1}, \nu\right)}{a\left(\gamma_{1}, \nu\right)}-\frac{a^{\prime \prime}\left(-\gamma_{1}, \nu\right)}{a\left(-\gamma_{1}, \nu\right)}-\left(\frac{a^{\prime}\left(\gamma_{1}, \nu\right)}{a\left(\gamma_{1}, \nu\right)}\right)^{2}+\left(\frac{a^{\prime}\left(-\gamma_{1}, \nu\right)}{a\left(-\gamma_{1}, \nu\right)}\right)^{2} .
$$

The error of the formula (28) does not exceed $(2 M \delta)^{1 / 2}$, see [3, p. 344]. To apply the theory of stable differentiation, an a priori upper bound $M$ for the absolute value of the second derivative of $f$ should be known or estimated.

In our simulation, the second derivative is calculated analytically in the form of the truncated series and its maximum is found to be $M=205$. The numerical results, based on this method, are presented in Section 3.2.

\subsection{Transformation to prolate coordinate system}

Let us consider the straight line passing through the origin of the coordinate system which is placed at the centre of symmetry of the large spheroid and the point $\xi$ 
of the small body:

$$
\begin{aligned}
& \left(x_{1}, y_{1}, z_{1}\right)=(0,0,0) \\
& \left(x_{2}, y_{2}, z_{2}\right)=\left(\xi_{1}+d_{1}, \xi_{2}+d_{2}, \xi_{3}+d_{3}\right) .
\end{aligned}
$$

In Equation (42), $d$ is the distance between the centres of the prolate spheroids. The components of the vector, joining the centres of the spheroids, are given by:

$$
\left\{\begin{array}{l}
d_{1}=d \sin \theta \cos \varphi, \\
d_{2}=d \sin \theta \cos \varphi, \\
d_{3}=d \cos \theta .
\end{array}\right.
$$

Equation of the straight line, joining the points $\left(x_{1}, y_{1}, z_{1}\right)$ and $\left(x_{2}, y_{2}, z_{2}\right)$, is given by

$$
\frac{x-x_{1}}{x_{2}-x_{1}}=\frac{y-y_{1}}{y_{2}-y_{1}}=\frac{z-z_{1}}{z_{2}-z_{1}} \text {. }
$$

Since $x_{1}=y_{1}=z_{1}=0$ this is equivalent to

$$
\frac{x}{x_{2}}=\frac{y}{y_{2}}=\frac{z}{z_{2}},
$$

where the point $(x, y, z)$ lies on the large prolate spheroid:

$$
\frac{x^{2}}{b_{1}^{2}}+\frac{y^{2}}{b_{1}^{2}}+\frac{z^{2}}{a_{1}^{2}}=1 .
$$

Using (43), one can express $x$ and $y$ as a function of $z, x_{2}, y_{2}, z_{2}$ :

$$
x=\frac{x_{2}}{z_{2}} z, \quad y=\frac{y_{2}}{z_{2}} z .
$$

Substituting (44) into (43), one gets

$$
z=\sqrt{\frac{z_{2}^{2}}{\left(x_{2}^{2} / b_{1}^{2}\right)+\left(y_{2}^{2} / b_{1}^{2}\right)+\left(z_{2}^{2} / a_{1}^{2}\right)}} .
$$

The scattering directions are: $\theta_{\xi}=\arccos \left(z / a_{1}\right), \varphi_{\xi}=\arctan \left(y_{2} / x_{2}\right), a_{2}=\left(z_{2} / \cos \theta_{\xi}\right)$ and $b_{2}=\left(x_{2} / \sin \theta_{\xi} \cos \varphi_{\xi}\right)$. The point $\xi$ in the prolate coordinate system is:

$$
\xi=\left(\cosh ^{-1}\left(\frac{a_{2}}{f}\right), \theta_{\xi}, \varphi_{\xi}\right)=\left(\zeta_{\xi}, \theta_{\xi}, \varphi_{\xi}\right),
$$

where $f:=\sqrt{a_{2}^{2}-b_{2}^{2}}$.

One can calculate $u_{1}(\xi, \alpha)$ by the formula

$$
u_{1}(\xi, \alpha)=u_{0}(\xi, \alpha)+u_{s}(\xi, \alpha),
$$

where

$$
u_{0}=e^{i k f\left(\sinh \zeta_{\xi} \sin \theta_{\xi} \cos \varphi_{\xi} \sin \widetilde{\alpha}+\cosh \zeta_{\xi} \cos \theta_{\xi} \cos \widetilde{\alpha}\right)}
$$


and

$$
\begin{aligned}
u_{s}= & -2 \sum_{m=0}^{\infty} \sum_{n=m}^{\infty} \epsilon_{m} \frac{i^{n}}{N_{m n}} \frac{R_{m n}^{(1)}\left(k f, \cosh \zeta_{1}\right)}{R_{m n}^{(3)}\left(k f, \cosh \zeta_{1}\right)} \\
& \times R_{m n}^{(3)}\left(k f, \cosh \zeta_{\xi}\right) S_{m n}(k f, \cos \widetilde{\alpha}) S_{m n}\left(k f, \cos \theta_{\xi}\right) \cos \left(m \varphi_{\xi}\right) .
\end{aligned}
$$

\section{Numerical simulation}

This section presents the simulation results for the two-body scattering problems. The spheroids considered have shape parameters that are of interest in practice. These examples simulate scattering by a submarine with $a_{1}=112, b_{1}=10$, and a small object near it, e.g. a mine or other object. Two methods are used for locating the position of the small body: solving the linear system (35) and calculating the derivative (27). In the subsections below the relative error for the calculated capacitance defined as:

$$
E_{\text {relat }}=100 \frac{\left|C_{\mathrm{obt}}-C_{\text {exac }}\right|}{C_{\mathrm{obt}}},
$$

and the estimated location of the small body are reported.

The numerical experiments consist of the following:

(1) The scattering amplitude $A_{2}$ is calculated by formula (11) and noise is added to the calculated values of $A_{2}: A_{2, \delta}=A_{2}+\delta n\left\|A_{2}\right\|$, where $\left\|A_{2}\right\|=\left\|A_{2}\right\|_{L^{2}\left(S^{2}\right)}$.

The noise $n$ is Gaussian, with mean value zero and standard deviation (std) equal to one. To simulate the corruption with Gaussian noise the Matlab pseudorandom generator for the normal distribution was used. The relative noise level $\delta$ is taken to be $0.01,0.03$ and 0.05 . The results are presented in the Sections 3.1 and 3.2.

The orientation of the small spheroid $D_{2}$ does not play a role in our numerics because the scalar wave scattering by a small acoustically soft body is isotropic. It will play a role if one generalizes our theory to the case of EM wave scattering, and also in the case of scalar wave scattering by acoustically hard small body [3, p. 99], formula (7.39).

(2) The ratio in the left side of formula (25) is calculated,

(3) The vector $\xi$ is calculated by solving linear system (33), and the capacitance $C$ is calculated by formula (28).

The quantity $u_{1}(\xi, \alpha)$ in the denominator of (28) is calculated by the formula $u_{1}=u_{0}+u_{s}$, where $u_{s}$ is calculated by formula (31).

\subsection{Solving linear systems}

To see the influence of the small body size, two cases with the small body of the sizes $a=0.5, b=0.25$ and $a=0.5, b=0.125$ are considered. The results for the first case are presented in Figure 1 and Table 1; and for the second case in Figure 2 and Table 2. Figures 1 and 2 show the error in the capacitance for different incident angles and for different levels of noise. As the noise level grows, then, for the smaller body (Case 2) the error grows almost linearly as a function of the noise level. Tables 1 and 2 show the 


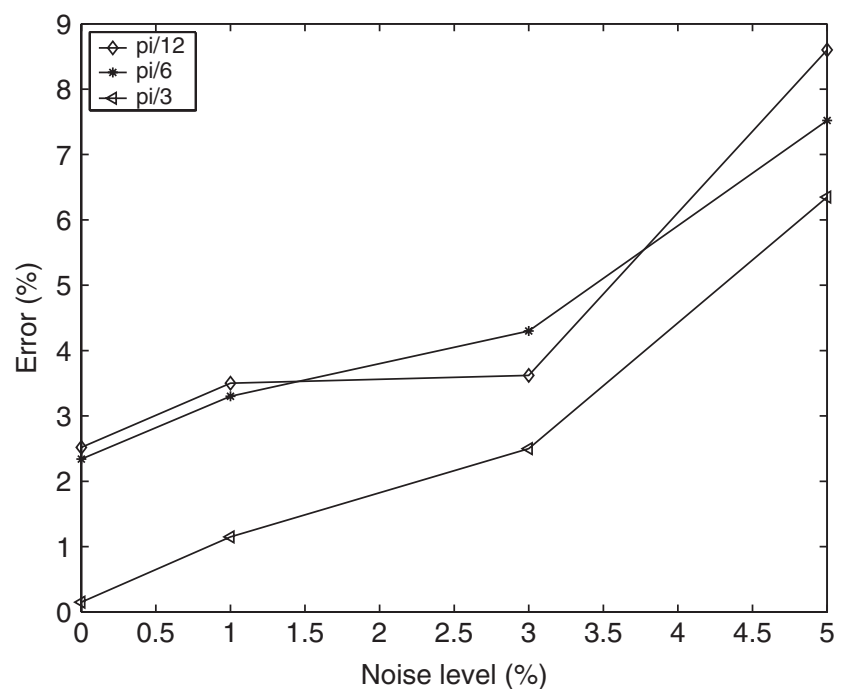

Figure 1. Relative error (45) for calculated capacitance. The results are given for $a=0.5, b=0.25$ (small prolate spheroid) and $a_{1}=112, b_{1}=10$ (large prolate spheroid) with noise. The estimated capacitance is calculated as $C=-4 \pi e^{i k \beta \cdot \xi} A_{2}(\beta, \alpha) / u_{1}(\xi, \alpha)$ and exact capacitance as $C=\sqrt{a^{2}-b^{2}} / \operatorname{arccosh}(a / b)$.

Table 1. The results (location of small body) for $a=0.5, b=0.25$ (small prolate spheroid) and $a_{1}=112, b_{1}=10$ (large prolate spheroid) for different levels of noise.

\begin{tabular}{lcccc}
\hline Incident angle & Noise level & $\xi_{1}$ & $\xi_{2}$ & $\xi_{3}$ \\
\hline$\frac{\pi}{12}$ & Without noise & 197.969581 & 194.544423 & 282.446045 \\
& $1 \%$ & 197.969995 & 194.544527 & 282.447164 \\
& $3 \%$ & 197.970766 & 194.544720 & 282.449254 \\
$\pi$ & $5 \%$ & 197.971447 & 194.544889 & 282.451103 \\
$\overline{6}$ & & 175.499492 & 175.500000 & 248.193465 \\
& Without noise & 175.500210 & 175.500104 & 248.194439 \\
& $1 \%$ & 175.501546 & 175.500296 & 248.196257 \\
$\pi$ & $5 \%$ & 175.502727 & 175.500465 & 248.197867 \\
$\overline{3}$ & $5 \%$ & 197.999120 & 198.000000 & 280.013699 \\
& Without noise & 198.000298 & 198.000104 & 280.014141 \\
& $1 \%$ & 198.002493 & 198.000296 & 280.014969 \\
& $3 \%$ & 198.004432 & 198.000465 & 280.015703 \\
\hline
\end{tabular}

calculated locations of the small body, for the two cases. The small body location change is of order $10^{-4}$ when the data are noisy and the noise level does not exceed 0.05 .

\subsection{Calculating the derivatives}

This section presents the results, similar to those reported in the previous section, but obtained by the stable differentiation method. 


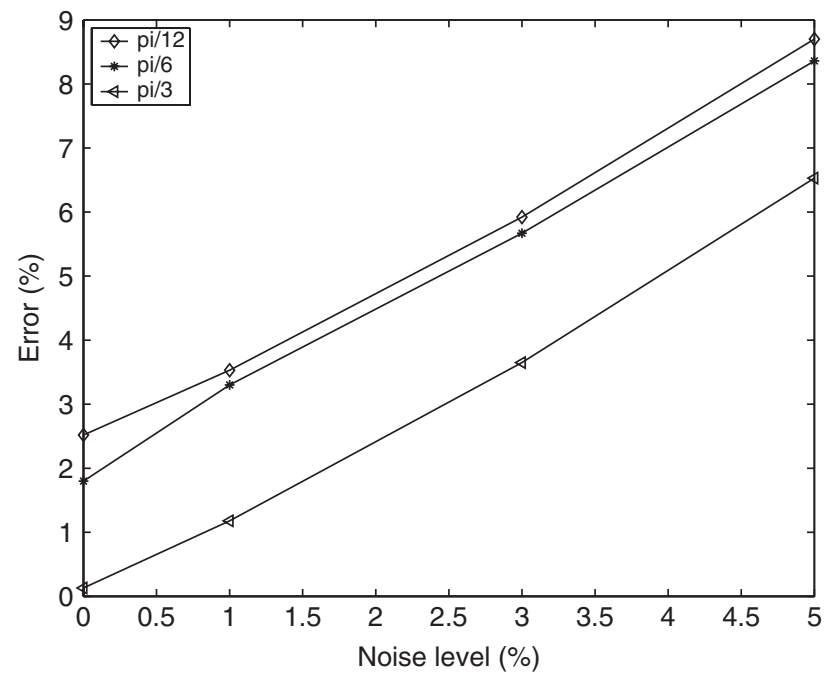

Figure 2. Relative error (45) for calculated capacitances. The results are given for $a=0.5, b=0.125$ (small prolate spheroid) and $a_{1}=112, b_{1}=10$ (large prolate spheroid) with noise. The estimated capacitance is calculated as $C=-4 \pi e^{i k \beta \cdot \xi} A_{2}(\beta, \alpha) / u_{1}(\xi, \alpha)$ and the exact capacitance as $C=\sqrt{a^{2}-b^{2}} / \operatorname{arccosh}(a / b)$.

Table 2. The results (location of small body) for $a=0.5, b=0.125$ (small prolate spheroid) and $a_{1}=112, b_{1}=10$ (large prolate spheroid) for different levels of noise.

\begin{tabular}{lcccc}
\hline Incident angle & Noise level & $\xi_{1}$ & $\xi_{2}$ & $\xi_{3}$ \\
\hline$\frac{\pi}{12}$ & Without noise & 197.969726 & 194.544423 & 282.446525 \\
& $1 \%$ & 197.970150 & 194.544682 & 282.447298 \\
& $3 \%$ & 197.970936 & 194.545161 & 282.448736 \\
$\pi$ & $5 \%$ & 197.971626 & 194.545580 & 282.450004 \\
$\overline{6}$ & Without noise & 175.499773 & 175.500000 & 248.193895 \\
& $1 \%$ & 175.500258 & 175.500258 & 248.194551 \\
& $3 \%$ & 175.501523 & 175.500737 & 248.195773 \\
$\pi$ & $5 \%$ & 175.502522 & 175.501156 & 248.196850 \\
$\frac{\pi}{3}$ & Without noise & 197.999606 & 198.000000 & 280.013947 \\
& $1 \%$ & 198.000506 & 198.000258 & 280.014177 \\
& $3 \%$ & 198.002177 & 198.000737 & 280.014605 \\
& $5 \%$ & 198.003646 & 198.001156 & 280.014984 \\
\hline
\end{tabular}

The results are presented in Tables 3 and 4, for the two cases of small bodies with different sizes.

Comparing these results with those, reported in the previous section (Table 3, last row for $\pi / 3$ ), we see that the two methods yield similar results.

\section{Summary}

The two-body scattering problem is studied. One of the bodies is small and the other one is large. The scattering problem by one large body, in the absence of the small one, is 
Table 3. The results (location of small body) for $a=0.5, b=0.25$ (small prolate spheroid) and $a_{1}=112, b_{1}=10$ (large prolate spheroid) and scattering direction $\pi / 3$ for different levels of noise.

\begin{tabular}{lcccc}
\hline & & \multicolumn{3}{c}{ Noise level } \\
\cline { 3 - 5 } Measurement & Without noise & $1 \%$ & $3 \%$ & $5 \%$ \\
\hline$\xi_{1}$ & 197.999828 & 198.999829 & 198.999825 & 198.999826 \\
$\xi_{2}$ & 197.999999 & 197.999999 & 198.999999 & 198.999999 \\
$\xi_{3}$ & 280.014285 & 280.014285 & 280.014286 & 280.014286 \\
Estimated capacitance & 0.329316 & 0.332541 & 0.31576 & 0.314542 \\
Exact capacitance & 0.328798 & 0.328798 & 0.328798 & 0.328798 \\
Error (\%) & 0.157 & 1.14 & 4.12 & 4.53 \\
\hline
\end{tabular}

Note: The estimated capacitance is calculated as $C=-4 \pi e^{i k \beta \cdot \xi} A_{2}(\beta, \alpha) / u_{1}(\xi, \alpha)$ and exact capacitance as $C=\sqrt{a^{2}-b^{2}} / \operatorname{arccosh}(a / b)$.

Table 4. The results (location of small body) for $a=0.5, b=0.125$ (small prolate spheroid) and $a_{1}=112, b_{1}=10$ (large prolate spheroid) and scattering direction $\pi / 3$ for different levels of noise.

\begin{tabular}{lcccc}
\hline & & \multicolumn{3}{c}{ Noise level } \\
\cline { 3 - 5 } Measurement & Without noise & $1 \%$ & $3 \%$ & $5 \%$ \\
\hline$\xi_{1}$ & 197.999897 & 197.999896 & 197.999898 & 198.999895 \\
$\xi_{2}$ & 197.999999 & 197.999999 & 197.999999 & 198.999999 \\
$\xi_{3}$ & 280.014285 & 280.014285 & 280.014285 & 280.014285 \\
Estimated capacitance & 0.234951 & 0.236416 & 0.243787 & 0.252190 \\
Exact capacitance & 0.234620 & 0.234620 & 0.234620 & 0.234620 \\
Error $(\%)$ & 0.141 & 0.7597 & 3.76 & 6.96 \\
\hline
\end{tabular}

Note: The estimated capacitance is calculated as $C=-4 \pi e^{i k \beta \cdot \xi} A_{2}(\beta, \alpha) / u_{1}(\xi, \alpha)$ and exact capacitance as $C=\sqrt{a^{2}-b^{2}} / \operatorname{arccosh}(a / b)$.

assumed solved. A method for solving the inverse problem of finding the location of the small body and the electric capacitance of the perfect conductor with the shape of the small body from the scattering data for the two-body scattering problem is presented. A good agreement between the numerical and the theoretical results is demonstrated.

\section{References}

[1] A.G. Ramm, Scattering by Obstacles, Reidel, Dordrecht, 1986.

[2] P.A. Martin, Multiple Scattering: Interaction of Time-Harmonic Waves with $N$ Obstacles, Encyclopedia of Mathematics and its Applications, Vol. 107, Cambridge University Press, Cambridge, 2006.

[3] A.G. Ramm, Wave Scattering by Small Bodies of Arbitrary Shapes, World Scientific Publishers, Singapore, 2005.

[4] — Dynamical Systems Method for Solving Operator Equations, Elsevier, Amsterdam, 2007.

[5] J.J. Bowman, T.B.A Senior, and P.L.E. Uslenghi, Electromagnetic and Acoustic Scattering by Simple Shapes, North-Holland Publishing Company, Amsterdam, 1969. 